\title{
Can computer simulators accurately represent the pathophysiology of individual COPD patients?
}

\author{
Wenfei Wang ${ }^{1 \dagger}$, Anup Das ${ }^{1 \dagger}$, Tayyba Ali $^{2}$, Oanna Cole ${ }^{2}$, Marc Chikhani ${ }^{2}$, Mainul Haque ${ }^{2}$, Jonathan G Hardman ${ }^{2}$ \\ and Declan G Bates $^{1 *}$
}

\author{
* Correspondence: \\ D.Bates@warwick.ac.uk \\ ${ }^{\dagger}$ Equal contributors \\ ${ }^{1}$ School of Engineering, University \\ of Warwick, Coventry CV4 7AL, UK \\ Full list of author information is \\ available at the end of the article
}

\begin{abstract}
Background: Computer simulation models could play a key role in developing novel therapeutic strategies for patients with chronic obstructive pulmonary disease (COPD) if they can be shown to accurately represent the pathophysiological characteristics of individual patients.
\end{abstract}

Methods: We evaluated the capability of a computational simulator to reproduce the heterogeneous effects of COPD on alveolar mechanics as captured in a number of different patient datasets.

Results: Our results show that accurately representing the pathophysiology of individual COPD patients necessitates the use of simulation models with large numbers (up to 200) of compartments for gas exchange. The tuning of such complex simulation models 'by hand' to match patient data is not feasible, and thus we present an automated approach based on the use of global optimization algorithms and high-performance computing. Using this approach, we are able to achieve extremely close matches between the simulator and a range of patient data including $\mathrm{PaO}_{2}, \mathrm{PaCO}_{2}$, pulmonary deadspace fraction, pulmonary shunt fraction, and ventilation/perfusion $(\dot{V} / \mathrm{Q})$ curves. Using the simulator, we computed combinations of ventilator settings that optimally manage the trade-off between ensuring adequate gas exchange and minimizing the risk of ventilator-associated lung injury for an individual COPD patient.

Conclusions: Our results significantly strengthen the credibility of computer simulation models as research tools for the development of novel management protocols in COPD and other pulmonary disease states.

Keywords: COPD; Computer simulation; Mechanical ventilation; Critical care medicine; Model matching; Global optimization

\section{Background}

Chronic obstructive pulmonary disease (COPD) is a leading cause of mortality and disability internationally and is predicted by 2020 to be the third most likely cause of death $[1,2]$. COPD is a progressive disease and is associated with increasing frequency and severity of exacerbations. Mechanical ventilation (MV), either invasive or noninvasive, may be a life-saving measure in managing respiratory failure due to an acute exacerbation of COPD [3]. However, mechanical ventilation is also associated with significant rates of morbidity and mortality. An improved understanding of the

\section{Springer}

(c) 2014 Wang et al.; licensee Springer. This is an Open Access article distributed under the terms of the Creative Commons Attribution License (http://creativecommons.org/licenses/by/4.0), which permits unrestricted use, distribution, and reproduction in any medium, provided the original work is properly credited. 
underlying pathophysiologic mechanisms of COPD is essential for the development of more effective and more individualized ventilation strategies for COPD patients.

Computer simulators that can accurately represent the particular disease state of an individual COPD patient could be an extremely valuable research tool for investigating the respiratory pathophysiology of COPD and predicting the effects of specific MV settings on the patient. Many researchers have worked on the development of physiological simulators, and various types of mathematical models have been proposed in the literature (e.g., [4-11]). However, these models generally employed only a very small number of compartments for gas exchange - in this paper, we show that such model cannot provide an accurate representation of the particular heterogeneous effects of COPD on alveolar mechanics. Also, most previous efforts to match physiological simulators to specific disease states have relied on manually manipulating the parameters in the simulator. Loeppky and co-workers [7] used a two-compartment model to match the COPD patient data generated from the multiple inert gas elimination technique (MIGET). Besides the use of a rather simplistic model, the study suffered from the use of only two parameters to represent $\mathrm{V} / \mathrm{Q}$ mismatching; this is a significant limitation, since gas exchange in COPD patients is often characterized by different $\dot{V} / Q$ patterns with two or three modes [12]. In more sophisticated multi-compartmental models, matching has been achieved with some success by adjusting the resistance for $100 \mathrm{com}$ partments manually [13], a challenging and time-consuming task. Indeed, it is obvious that manual matching is only practical for relatively simple models with a small set of adjustable parameters and a limited set of patient data for matching.

A small number of previous studies have investigated the use of numerical optimisation approaches for the model matching task; a tidally breathing model has been matched to MIGET measurements from emphysema and embolism patients using the interior-reflective Newton algorithm [14], and two and three compartmental models were matched to data from intensive care patients with acute lung injury (ALI) using Brent's method [15]. These studies again used rather simple models with only a few compartments, and both studies employed very simple optimization algorithms, for which the quality of the matching achieved depended completely on the initial values chosen for the optimisation parameters. Since there is very little information available about how to choose these initial estimates, the likelihood of such approaches finding the best possible match to patient data is very small. Moreover, due to the simplified nature of the models used, continuous $\dot{\mathrm{V}} / \mathrm{Q}$ distributions could not be produced, and thus, the model outputs can only be compared to patient data at two or three isolated points on the $\dot{V} / Q$ curve.

In this paper, we investigate the minimum level of complexity required in a computer simulation model in order to provide a representation of COPD pathophysiology that is accurate enough to allow the simulator to be used for studies on the design of novel therapeutic strategies for individual patients. In order to address this question, we employ a sophisticated simulation model of lung physiology that incorporates tidal ventilation, pulsatile pulmonary blood flow, hypoxic pulmonary vasoconstriction (HPV), a realistic and validated oxygen-hemoglobin model, and up to 200 individually configurable alveolar compartments. Our simulator has been developed over the past decade and has been used and validated in a number of previous studies $[16,17,13]$. In the present study, we couple this simulator to software implementing a global optimization 
algorithm [18], based on evolutionary principles (a genetic algorithm) which allows large numbers of model parameters to be simultaneously optimized in order to match the model outputs to detailed COPD patient data. An attractive feature of our approach from the point of view of clinical researchers and practitioners is that it can be highly automated, so that the user does not need to have detailed knowledge of the underlying algorithms but can use the software to fit models of varying complexity to different patient data sets. To illustrate how the proposed simulator could be used to investigate questions of clinical relevance, we present the results of an investigation into computing ventilator settings that optimally manage the trade-off between ensuring adequate gas exchange and minimizing the risk of ventilator-associated lung injury for an individual COPD patient.

\section{Methods}

\section{The computational simulator}

The simulation used in this study is a multi-compartmental computational model that uses an iterative technique to simulate integrated respiratory and cardiovascular pathophysiological scenarios $[17,19,20]$. A detailed description of the principles and mathematical equations underlying the computational model implemented in our simulator is provided in Additional file 1. In contrast to previous models of COPD pathophysiology that included only two or three alveolar compartments, our model allows the user to define the number of compartments (each with its own individual mechanical characteristics) to be implemented in the simulation. This allowed us to investigate in detail the relationship between the number of compartments in the model and its ability to match individual patient data. Each $i$ th alveolar compartment has a unique and configurable bronchiolar resistance $R_{B, i}$ [ $\left.\mathrm{cmH}_{2} \mathrm{O} \cdot \mathrm{s} / \mathrm{l}\right]$, pulmonary vascular resistance $R_{V, i}$ [dyn.s $\left./ \mathrm{cm}^{5}\right]$, stiffness index $S_{i}\left[\mathrm{cmH}_{2} \mathrm{O} / \mathrm{ml}^{2}\right]$, and extrinsic pressure $P_{\text {ext, } \mathrm{i}}\left[\mathrm{cmH}_{2} \mathrm{O}\right]$ (giving $4 \times \mathrm{N}$ adjustable parameters in total, where $N$ is the number of compartments). The ability to adjust these parameters individually across up to 200 alveolar compartments allows the model to recreate the heterogeneous effects of COPD on the overall physiology of the lung. The model also includes specific equations to represent the effects of alveolar collapse, threshold opening pressure, alveolar stiffening, and airway obstruction. The net effect of these components of the simulation is that the defining, clinical features of COPD may be observed in the model: alveolar gas trapping (with intrinsic positive end-expiratory pressure (PEEP)), collapse-reopening of alveoli (with gradual reabsorption of trapped gas if reopening does not occur), limitation of expiratory flow, and increased functional residual capacity - see Additional file 1 for further details.

\section{Patient data}

Two different sets of patient data from the literature are used in this study. The first dataset is from [21], where a 55-year-old patient is sedated and paralyzed, and relevant data for configuring the model and ventilator settings are shown in Table 1. For this case, we attempt to match the outputs of our simulator to the data reported for the following patient variables - $\mathrm{PO}_{2}, \mathrm{PCO}_{2}$, deadspace, shunt, mean and standard deviation of $\dot{\mathrm{V}}$ and $\mathrm{Q}$, and ventilation-perfusion distribution across a number of ranges 
Table 1 Model configuration for the first patient dataset

\begin{tabular}{lc}
\hline Parameter & Value \\
\hline Respiratory frequency $[\mathrm{bpm}]$ & 13 \\
Tidal volume $[\mathrm{ml}]$ & 590 \\
$\mathrm{FIO}_{2}$ & 0.4 \\
Inspiratory flow pattern & Constant flow \\
Cardiac output $[\mathrm{l} / \mathrm{min}]$ & 5.0 \\
PEEP $\left[\mathrm{cmH}_{2} \mathrm{O}\right]$ & 0 \\
$\mathrm{IE}$ & $1: 3$ \\
$\mathrm{RQ}$ & 0.8 \\
\hline
\end{tabular}

(see Table 2). The second set of patient data is from [12]. The 64-year-old patient is reported to be in a stable condition, and relevant model and ventilator configuration parameters are shown in Table 3. For this case, we attempt to match our model directly to patient $\dot{V} / Q$ curves generated via MIGET measurements.

\section{Automated matching to patient data}

Exacerbations of COPD are frequently associated with deterioration in gas exchange and associated hypoxemia. Unsurprisingly, increased inequality in $\dot{\mathrm{V}} / \mathrm{Q}$ relationships appears to be the major determinant of these changes [1]. Therefore, a key requirement for the simulation of COPD pathophysiology is the ability to accurately match the $\dot{V} / \mathrm{Q}$ distributions seen in patient data. In our simulator, the $\dot{\mathrm{V}} / \mathrm{Q}$ distribution can be manipulated by adjusting the bronchiolar resistance and pulmonary vascular resistance, stiffness, and extrinsic pressure for each compartment in the model. For example, by increasing vascular resistance in a region of the lung, an area of relative dead space can be created. While all parameters can be manually adjusted, this becomes impractical as the number of compartments $N$ in the model increases, since the total number of parameters is $4 \times \mathrm{N}$.

To address this issue, we formulate the model-matching problem as an optimization problem, where the difference between the model outputs and the data is captured in a cost function, and the model parameters that can be varied are the variables for the optimization problem. As mentioned above, two sets of patient data are considered in this study. For both cases, a primary focus is on accurately representing the imbalance in the $\dot{V} / Q$ distribution caused by the disease state. In the first dataset [21], the horizontal axis of the $\dot{V} / Q$ diagram is divided into several segments on which the percentage of $\dot{V} / Q$ indicated in the data will be matched. Other parameters that also need to be matched include $\mathrm{PaO}_{2}, \mathrm{PaCO}_{2}$, and mean and standard deviation of ventilation and perfusion. For the second case, the whole $\dot{V} / \mathrm{Q}$ curve is considered (i.e., every point on the curve is to be matched). The matching error can then be defined based on these data, which is given as:

$$
E_{T}=\sum_{i=1}^{n} E_{i}^{2}
$$

where $E_{T}$ is the total residual error representing the matching accuracy, $E_{i}=\frac{x_{i}-x_{i d}}{x_{i d}}$ is the error for parameter $i, x_{i}$ is the model output value for parameter $i$, and $x_{i d}$ is the value of the data for parameter $i$. 
Table 2 Matched parameter values and the reference data for the first patient dataset

\begin{tabular}{|c|c|c|c|c|c|c|}
\hline \multirow[t]{2}{*}{ No. } & \multirow[t]{2}{*}{ Parameter } & \multirow[t]{2}{*}{ Data } & \multicolumn{4}{|c|}{ Model outputs ( $N=$ number of compartments) } \\
\hline & & & $N=10$ & $N=25$ & $N=50$ & $N=100$ \\
\hline 1 & $\mathrm{PaO}_{2}[\mathrm{~mm} \mathrm{Hg}]$ & 125.2 & 165.29 & 143.18 & 133.73 & 127.92 \\
\hline 2 & $\mathrm{PaCO}_{2}[\mathrm{~mm} \mathrm{Hg}]$ & 46 & 43.08 & 44.73 & 43.62 & 45.76 \\
\hline 3 & Dead space fraction & 64.9 & 61.24 & 62.76 & 61.28 & 65.28 \\
\hline 4 & Shunt fraction & 6.8 & 12.9 & 9.02 & 8.07 & 7.2 \\
\hline 5 & mean_过 & 0.99 & 1 & 1 & 1 & 1 \\
\hline 6 & mean_Q & 0.27 & 0.2 & 0.2 & 0.25 & 0.2 \\
\hline 7 & sd_d & 1 & 0.92 & 1.15 & 0.92 & 1.15 \\
\hline 8 & sd_Q & 1.34 & 1.38 & 1.38 & 1.38 & 1.38 \\
\hline 9 & $0.1<\dot{\mathrm{V}} / \mathrm{Q}<1, \dot{\mathrm{V}}$ & 19.9 & 20.43 & 20.06 & 22.68 & 19.94 \\
\hline 10 & $1<\dot{V} / Q<10, \dot{V}$ & 14.3 & 19.69 & 16.57 & 16.47 & 14.48 \\
\hline 11 & $0.01<\dot{V} / Q<0.1, P$ & 15.8 & 22.63 & 18.86 & 14.64 & 16.16 \\
\hline 12 & $0.1<\dot{V} / Q<1, P$ & 62.1 & 55.21 & 58.12 & 65.57 & 64.92 \\
\hline \multirow[t]{3}{*}{13} & $1<\dot{V} / Q<10, P$ & 10.9 & 11.05 & 11.58 & 10.63 & 10.56 \\
\hline & Total matching error & & 1.33 & 0.46 & 0.11 & 0.10 \\
\hline & Simulation time [h] & & 11 & 32 & 41 & 67 \\
\hline
\end{tabular}

Global optimization algorithms can then be used to find model parameter values that minimize the value of $E_{T}$, i.e., minimize the difference between the model outputs and the data. The procedure is illustrated in Figure 1 - in each iteration, a set of parameter combinations are sent to the simulator, and the outputs from the simulator are evaluated by the optimization algorithm which then generates the updated parameter values for the next iteration until the termination criterion is reached (i.e., the condition to get a best matching is found).

In the model-matching procedure, the model parameters are allowed to vary continuously between physiologically realistic upper and lower bounds - see Table 4. COPD is characterized by restrictions to flow in airways (bronchi and bronchioles) due to increased excretion of mucus and inflammation of the bronchiolar walls, effectively reducing the radius of the tube through which the airways ventilate the alveoli. Based on the Hagen-Pouiselle relationship:

$$
R \propto \frac{\eta L}{r^{4}}
$$

Table 3 Model configuration for the second patient dataset

\begin{tabular}{lc}
\hline Parameter & Value \\
\hline Respiratory frequency [bpm] & 16 \\
Tidal volume $[\mathrm{ml}]$ & 410 \\
$\mathrm{FiO}_{2}$ & 0.21 \\
Inspiratory flow pattern & Constant flow \\
Cardiac output [l/min] & 3.4 \\
PEEP $\left[\mathrm{CmH}_{2} \mathrm{O}\right]$ & 0 \\
$\mathrm{IE}$ & $1: 3$ \\
$\mathrm{RQ}$ & 0.8 \\
\hline
\end{tabular}




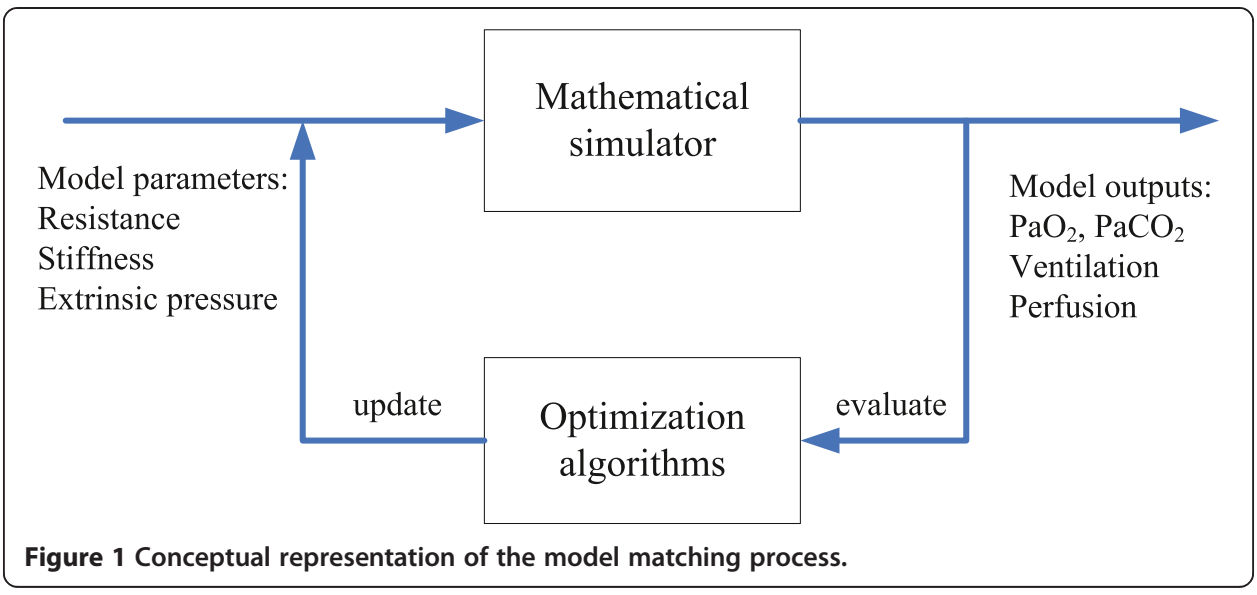

the resistance $(R)$ in a single tube is directly proportional to the length $(L)$ of the tube and the viscosity $(\eta)$ and inversely proportional to the fourth power of the radius $\left(r^{4}\right)$. To achieve the required precision in matching the extreme ranges of $\dot{V} / Q$ distributions for the second dataset, we have investigated the effect of increasing the upper bound on compartmental resistances from 500 to 2,000 times their nominal values, i.e., the radius of the airways can be reduced by as much as $85 \%$. To ensure that this does not result in model configurations that produce unrealistic values for total lung resistance, we incorporated a constraint function into the optimization algorithm that limits the increase in the total lung inflow resistance to ten times its basic (healthy) value, as expected in patients with COPD [22].

Previous attempts to use optimization for matching models of pulmonary disease states to patient data have used rather simple algorithms that require good initial 'guesses' for the parameters in order to be effective. In the case of COPD, however, current understanding of the associated pathophysiology provides little guidance into how to choose initial values for the model parameters across large numbers of alveolar compartments. In this study, we therefore employed an advanced global optimisation algorithm known as a genetic algorithm. This general purpose stochastic search and optimization procedure, based on genetic and evolutionary principles [23], has been shown to have a much higher chance of finding optimal solutions for difficult problems with large numbers of variables. Full details of the particular optimization algorithm used in this study and how it was implemented with the model are provided in Additional file 1.

\section{Using the simulator for clinical investigations}

To illustrate how the simulator can be used for clinical investigations, we consider the problem of identifying ventilator settings that minimize the risk of ventilator-associated lung injury (VALI). We consider the following five key ventilator settings as variable parameters that may be adjusted to optimize the trade-off between effective gas exchange and minimizing the risk of VALI: (1) tidal volume (Vtidal, [ml]) - the volume of air traveling in or out of the patient's lungs during every breath; (2) ventilation rate (VentRate, [breaths/min]) - the number of breaths per minute; (3) duty cycle (I:E) - the ratio of inspiratory time to total ventilatory cycle duration; (4) PEEP, $\left[\mathrm{cmH}_{2} \mathrm{O}\right]$ - the positive pressure in the lungs at the end of exhalation; and (5) fraction of inspired oxygen $\left(\mathrm{FIO}_{2}\right)$ - the fraction of oxygen constituting the inhaled volume of gas as provided by the mechanical ventilator. 
Table 4 Model parameters - nominal values and allowable ranges (for $N=100$ )

\begin{tabular}{lcc}
\hline Parameters & Nominal value & Variation ranges \\
\hline Bronchiolar resistance $R_{B i}\left[\mathrm{cmH}_{2} \mathrm{O} \cdot \mathrm{s} / \mathrm{l}\right]$ & 600 & {$\left[300,3 \times 10^{5}\right]$} \\
Vascular resistance $R_{V i}\left[\mathrm{dyn} \cdot \mathrm{s} / \mathrm{cm}^{5}\right]$ & $1.6 \times 10^{4}$ & {$\left[8 \times 10^{3}, 8 \times 10^{6}\right]$} \\
Stiffness coefficient $S_{i}\left[\mathrm{cmH}_{2} \mathrm{O} / \mathrm{ml}^{2}\right]$ & 0.05 & {$[0.025,0.15]$} \\
Extrinsic pressure $P_{\text {ext,i }}\left[\mathrm{cm} \mathrm{H}_{2} \mathrm{O}\right]$ & 28.8 & {$[-20,28.8]$} \\
\hline
\end{tabular}

The maximum allowable ranges of variation for the values of these parameters have been defined based on current clinical practice and to be consistent with data available from clinical trials [24,25]. Vtidal is allowed to vary within a range from 390 to $650 \mathrm{ml}$, corresponding to 6 to $10 \mathrm{ml} / \mathrm{kg}$ for a body weight of $65 \mathrm{~kg}$. VentRate is bounded within the range 9 to 16 breaths/min, I:E is limited to the interval 0.25 to 0.5 (i.e., a ratio between 1:4 and 1:2), PEEP is constrained within 0 to $5 \mathrm{cmH}_{2} \mathrm{O}$, and $\mathrm{FIO}_{2}$ is bounded within 0.21 to 1 . A summary is provided in Table 5 .

Three key physiological indicators are also defined. To monitor effective arterial oxygenation, partial pressure of oxygen, $\mathrm{PaO}_{2}$, needs to be considered. In order to maintain effective arterial oxygenation, $\mathrm{PaO}_{2}$ is constrained to be higher than $8 \mathrm{kPa}$, with a desired value of $12 \mathrm{kPa}$. Arterial partial pressure of carbon dioxide, $\mathrm{PaCO}_{2}$ is another key indicator of alveolar ventilation that also indirectly reflects acid-base balance. $\mathrm{PaCO}_{2}$ is constrained to be between 4 and $8 \mathrm{kPa}$ with a desired value of $5.3 \mathrm{kPa}$. The risk of barotrauma is proportional to the peak alveolar pressure, (Palv, $\mathrm{kPa}$ above atmospheric pressure), and Palv is limited to $4 \mathrm{kPa}$, where Palv is calculated as the average of the peak pressure in the most highly pressurized $25 \%$ of all alveoli.

Requirements on the above physiological indicators can be captured as an optimization problem and formulated mathematically as:

$$
\min \left\{J_{1}, J_{2}\right\}
$$

where

$$
\begin{aligned}
& J_{1}=w_{1}\left|\mathrm{PaO}_{2}-12\right|+w_{2}\left|\mathrm{PaCO}_{2}-5.3\right| \\
& J_{2}=w_{3} P_{\text {alv }}
\end{aligned}
$$

\begin{tabular}{|c|c|c|}
\hline & \multicolumn{2}{|c|}{ Variation ranges } \\
\hline & Acceptable values & Desired \\
\hline \multicolumn{3}{|c|}{ MV setting parameters } \\
\hline Vt [ml] & {$[390,650]$} & \\
\hline VentRate [bpm] & {$[9,16]$} & \\
\hline l:E & {$[0.25,0.5]$} & \\
\hline PEEP $\left[\mathrm{cmH}_{2} \mathrm{O}\right]$ & {$[0,5]$} & \\
\hline $\mathrm{FiO}_{2}$ & {$[0.21,1]$} & \\
\hline \multicolumn{3}{|l|}{ Model outputs } \\
\hline $\mathrm{PO}_{2}[\mathrm{kPa}]$ & $>8$ & 12 \\
\hline $\mathrm{PCO}_{2}[\mathrm{kPa}]$ & $>4,<8$ & 5.3 \\
\hline Palv [kPa] & $<4$ & - \\
\hline
\end{tabular}

Table 5 MV setting parameter variation bounds and desired model outputs 
Large values of $J_{1}$ will be produced by ventilator settings that provide poor gas exchange, while large values of $J_{2}$ will be produced by combinations of ventilator settings that cause high peak alveolar pressures (and hence increase the risk of VALI). By requiring both $J_{1}$ and $J_{2}$ to be minimized simultaneously, we can search for combinations of ventilator settings that optimally manage the trade-off between effective gas exchange and minimizing the risk of VALI. $\mathrm{w}_{1}, \mathrm{w}_{2}$, and $\mathrm{w}_{3}$ are weighting functions that are used in the optimization process to ensure that equal priority is given to each of the different objectives. Since we are trying to minimize two objectives $J_{1}$ and $J_{2}$ at the same time, a multi-objective optimization algorithm called non-dominated sorting genetic algorithm II (NSGA-II) was used here [26].

\section{Results}

\section{Matching results for the first dataset}

For the first dataset, the ventilation-perfusion distribution is characterized by several segments based on the $\dot{V} / Q$ ratio, which includes the amount of ventilation in the ranges $0.1<\dot{\mathrm{V}} / \mathrm{Q}<1$ and $1<\dot{\mathrm{V}} / \mathrm{Q}<10$, and the amount of perfusion in the ranges $0.01<$ $\dot{\mathrm{V}} / \mathrm{Q}<0.1,0.1<\dot{\mathrm{V}} / \mathrm{Q}<1$, and $1<\dot{\mathrm{V}} / \mathrm{Q}<10$ together with the shunt and dead space. Other data considered are the $\mathrm{PO}_{2}, \mathrm{PCO}_{2}$, mean $\dot{\mathrm{V}} / \mathrm{Q}$ ratio of the pulmonary blood flow, and ventilation distribution as well as standard deviations (dispersion) of pulmonary blood flow and ventilation. The simulation was performed separately for four cases with the total number of compartments in the model being varied between 10, 25, 50, and 100 to represent different levels of model complexity.

Figure 2 and Table 2 report the fitted results for each scenario. It can be seen that incorporating a larger total number of compartments into the model significantly improves its ability to accurately represent the patient data, with the total fitting error reduced from 1.33 for 10 compartments to 0.46 for 25 compartments, to 0.11 for 50 compartments. Interestingly, when the total compartment number is increased from 50 to 100 , the fitting error only reduces from 0.11 to 0.10 , indicating that for this

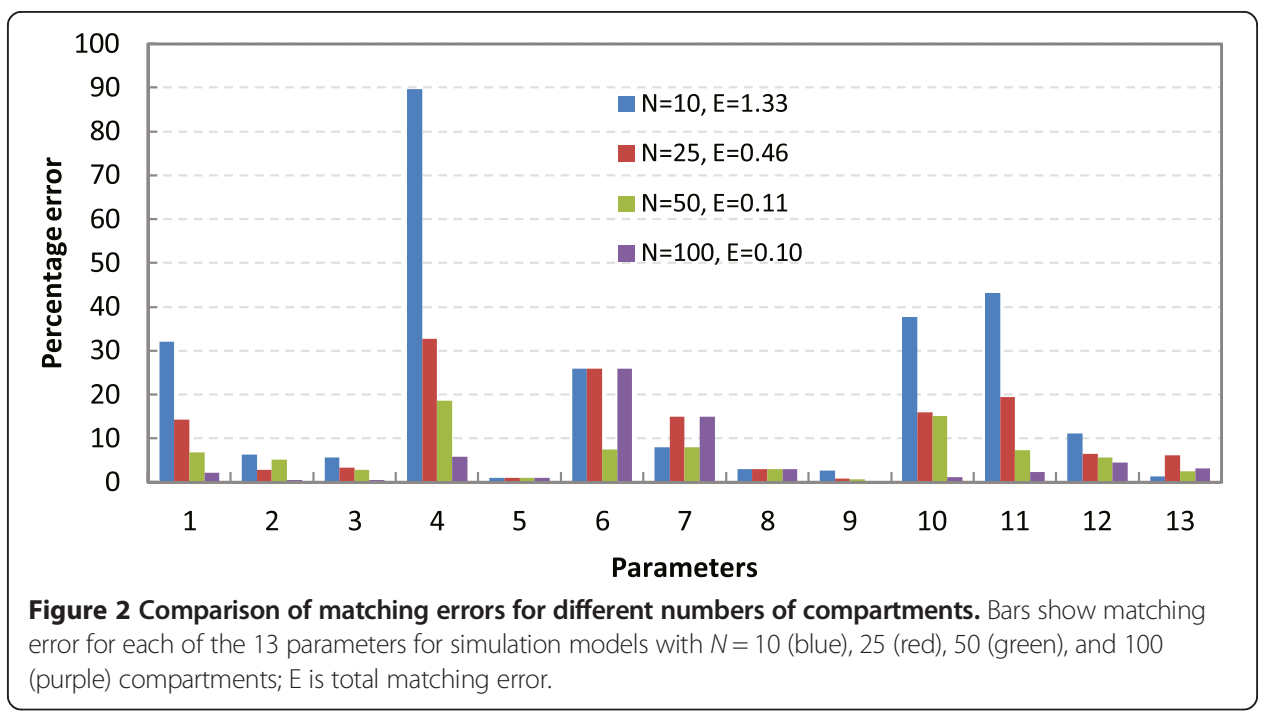


particular dataset, 50 compartments represent an adequate level of complexity. Considering that the computational burden associated with the parameter optimization rises proportionally with the complexity of the model, the ability to identify just how complex a model needs to be is clearly of primary importance for this type of problem.

\section{Matching results for the second dataset}

In the second dataset, we attempt to reproduce in our model the complete $\mathrm{V} / \mathrm{Q}$ curve generated from MIGET results [12]. Forty evenly distributed points are selected along the logarithm axis from 0.01 to 100 with each point representing an equal small segment. The ventilation and perfusion at each point are calculated by summing up the ventilation and perfusion of all alveoli with $\dot{V} / \mathrm{Q}$ ratio that falls into the respective segment. The values at each point are then compared with the data, and the total error was calculated based on Equation 1. An initial minimum number of 50 compartments were used in the model, based on the results of the previous dataset reported above. However, as shown in Figure 3a, with this number of compartments, a large discrepancy is observed between the data and best model fit (total fitting error 5.68). When the number of compartments is increased to 100 and 200, however, it can be seen from Figure 3c,e that the ability of the model to represent the V/Q curve improves, with the fitting error reducing to 4.46 and 3.91, respectively. To improve the model fit over the left part of the $\dot{\mathrm{V}} / \mathrm{Q}$ curve, higher bounds on the resistance variation $\bar{R}$ were then allowed in the optimization procedure. Figure $3 \mathrm{~b}$ shows that, for 50 compartments, the $\dot{V} / \mathrm{Q}$ curve in the range of 0.1 to 3 is matched much more closely after using a higher upper bound for the resistance, and the total matching error $\mathrm{E}$ is reduced from 5.68 to 4.25. Increasing the number of compartments to 100 and 200 further reduces the matching error to 3.24 and 2.80, respectively, so that, as shown in Figure 3f, a very accurate representation of the total $\dot{V} / Q$ curve can be produced by the model. Note that, for the higher upper bound on the resistances, the use of a constraint function in the optimization algorithm to ensure that the total lung resistance stays within physiologically reasonable values for COPD patients results in a doubling of the overall computation time required for the optimization to converge.

\section{Computation of optimal ventilation strategies using the simulator}

Table 6 compares the optimal MV settings computed using our simulator with those provided in the first patient dataset [21]. Figure 4 shows the so-called Pareto front returned by the NSGA-II algorithm. Each point on the Pareto front corresponds to a combination of ventilator settings for which any improvement in one objective function (e.g., gas exchange) necessarily results in a deterioration in the other (e.g., peak alveolar pressure). Thus, the Pareto front defines the trade-off inherent in the problem and allows us to investigate how different combinations of ventilator settings manage that trade-off. The optimal settings reported in Table 6 correspond to point No. 1 in Figure 4 and provide the most balanced solution to the two competing objectives. Compared with the data values, the peak alveolar pressure has been reduced from $5.1 \mathrm{kPa}$ to 3.8 $\mathrm{kPa}$, so that it is now within the maximum specified value of $4 \mathrm{kPa}$. This improvement has been achieved at the cost of a small increase in $\mathrm{PaCO}_{2}$ (from 6.1 to $6.3 \mathrm{kPa}$ ), while the value of $\mathrm{PaO}_{2}(12.2 \mathrm{kPa})$ is now very close to its desired value of $12 \mathrm{kPa}$. This 

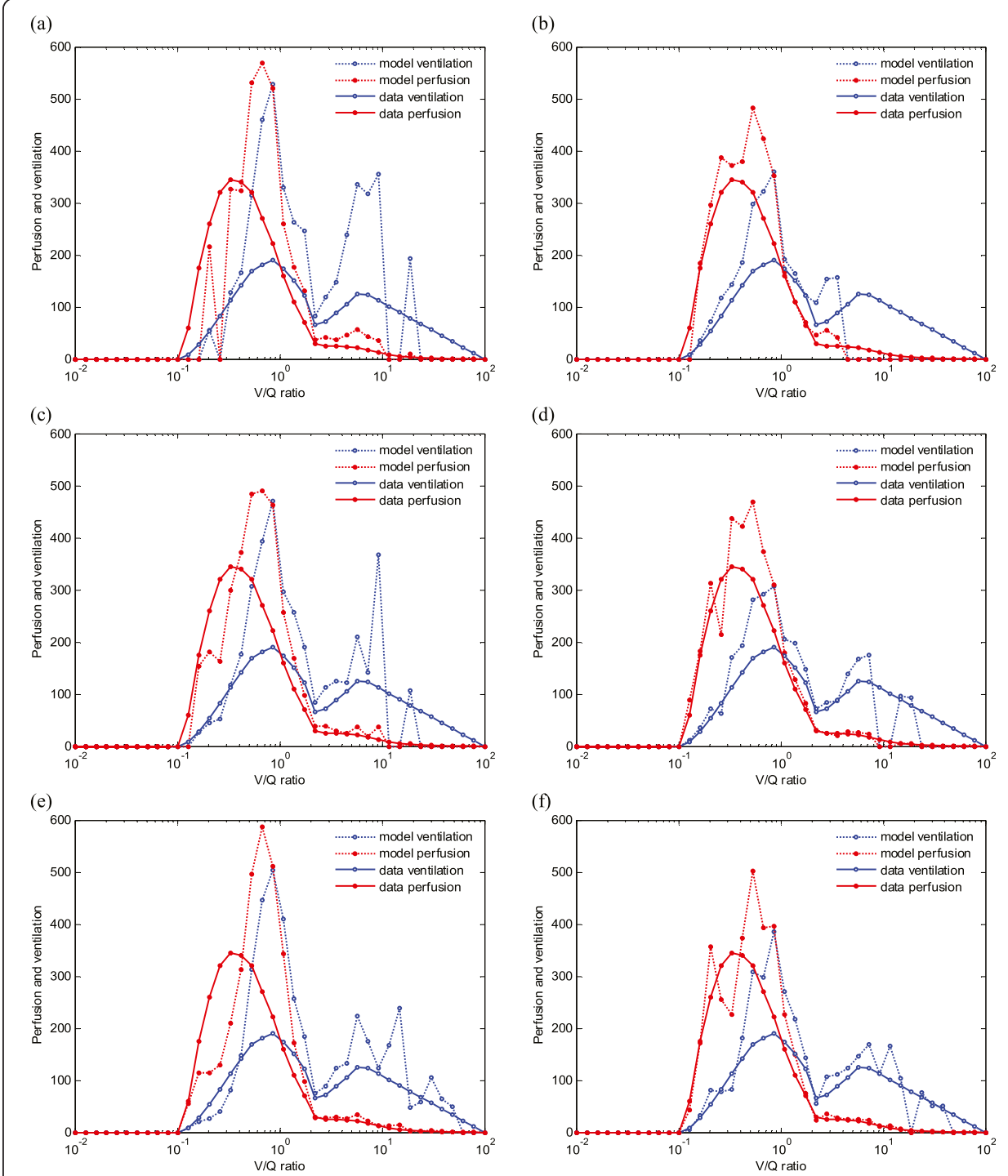

Figure 3 Simulated $\dot{V} / Q$ distribution compared with the data. $N$ is the number of compartments used, $\bar{R}_{B i}$ denotes upper bounds of bronchiolar resistance, and $\bar{R}_{V i}$ denotes upper bounds of vascular resistances for the ith compartment. (a) $N=50, \bar{R}_{B i}=3 \times 10^{5}, \bar{R}_{V i}=8 \times 10^{6}$, Error $=5.68$. (b) $N=50, \bar{R}_{B i}=1.2 \times 10^{6}$, $\bar{R}_{V i}=3.2 \times 10^{7}$, Error $=4.25$. (c) $N=100, \bar{R}_{B i}=3 \times 10^{5}, \bar{R}_{V i}=8 \times 10^{6}$, Error $=4.46$. (d) $N=100, \bar{R}_{B i}=1.2 \times 10^{6}$, $\bar{R}_{V i}=3.2 \times 10^{7}$, Error $=3.24$. (e) $N=200, \bar{R}_{B i}=3 \times 10^{5}, \bar{R}_{V i}=8 \times 10^{6}$, Error $=3.91$. (f) $N=200, \bar{R}_{B i}=1.2 \times 10^{6}$, $\overline{\mathrm{R}}_{\mathrm{Vi}}=3.2 \times 10^{7}$, Error $=2.80$.

'rebalancing' of the ventilator settings in favor of a more lung protective strategy has been achieved principally by reducing tidal volume from 590 to $506 \mathrm{ml}$, while simultaneously increasing the ventilation rate from 13 to $15 \mathrm{bpm}$. Changes in the other three ventilator settings are more modest, indicating that they have a relatively smaller influence on the chosen physiological indicators for this patient. Moving along the curve shown in Figure 4, different combinations of ventilator settings are computed which place more emphasis on one objective or the other. Point No. 2, for example, corresponds to a maximally protective strategy, where Palv has been further reduced to $2.8 \mathrm{kPa}$, at the cost of increasing $\mathrm{PaCO}_{2}$ to $7.4 \mathrm{kPa}$ and reducing $\mathrm{PaO}_{2}$ to 11.9 (note that both $\mathrm{PaCO}_{2}$ and $\mathrm{PaO}_{2}$ are still within their specified limits). 
Table 6 Optimal MV settings and model outputs compared with first patient dataset

Data

MV setting parameters

Vt [ml]

I:E

PEEP $\left[\mathrm{CmH}_{2} \mathrm{O}\right]$

$\mathrm{FiO}_{2}$

Model outputs

$\mathrm{PaO}_{2}[\mathrm{kPa}]$

$\mathrm{PaCO}_{2}[\mathrm{kPa}]$

Palv $[\mathrm{kPa}]$
VentRate $[b p m]$

$\begin{array}{cc}590 & 506 \\ 13 & 15 \\ 0.33 & 0.32 \\ 0 & 1 \\ 0.4 & 0.3 \\ & \\ 17 & 12.2 \\ 6.1 & 6.3 \\ 5.1 & 3.8\end{array}$

\section{Discussion and conclusions}

The high mortality and morbidity associated with COPD will make the condition a critical health burden for the future, particularly if current trends are allowed to continue $[27,28]$. The main treatment option for critical exacerbations of COPD remains mechanical ventilation (non-invasive and invasive) and for long-term management, oxygen therapy [25]. Prognosis of COPD is worsened with underlying respiratory failures, adding greatly to the cost of treating the disease [29,30]. Research into the condition using animal models of lung injury and in vitro experiments have failed to show a clear way forward, and large-scale clinical trials to monitor long-term effects in this fragile population are not possible $[31,6]$.

Computer simulations [6,32] offer a potentially powerful alternative platform for research into the development of new treatment strategies, but no previous studies have been able to demonstrate adequate matching to the particular disease characteristics of

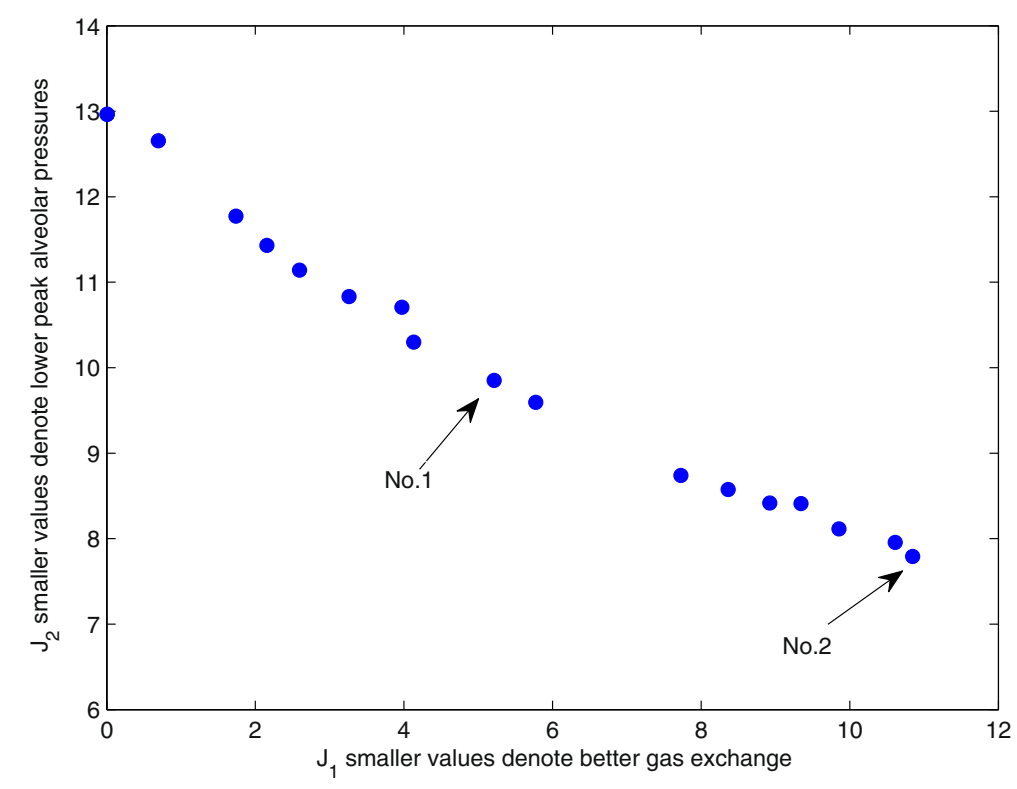

Figure 4 Pareto front from the multi-objective optimization. Shows the tradeoff between maximizing gas exchange and reducing the risk of VALI. 
COPD. In this study, we have described a computer simulation model that can accurately simulate an individual COPD patient receiving MV. Our study has focused on single patient subjects described in the literature, and the two studies that have been selected represent the spectrum of COPD cases that are found in clinical practice. Our results clearly show the importance of employing mathematical models of sufficient complexity to allow an accurate representation of disease states in individual patients. In particular, the heterogeneous effects of COPD on lung alveoli in different regions of the lung make it essential that large numbers of compartments are included in the model - for all datasets considered, matching between the simulation outputs and patient data was significantly improved as the number of alveolar compartments in the model was increased.

The need for a large number of compartments with heterogeneous dynamics creates an additional challenge - how to choose appropriate values for the large number of corresponding model variables. Indeed, a second major conclusion of our study is that 'manual' tuning of such parameters is simply not feasible for models of the complexity required. Instead, we have shown how global optimization algorithms, implemented using sophisticated parallel processing protocols, can allow optimal values of very large numbers of parameters to be identified. A significant advantage of this approach is that it can be largely automated, with the clinical researcher needing only to input the patient data and the simulation platform taking care of all other aspects of the modelmatching process.

Due to the nature of COPD, multiple comorbidities are associated with the symptoms and development of COPD including inflammation and heart and kidney dysfunctions. This presents a unique challenge to the clinician in terms of maintaining the correct balance between raising pressures and delivering oxygen, while keeping hypercapnea and hypoxia to a minimum [33,34]. The clinician needs to monitor multiple aspects of physiology simultaneously, and many adverse effects are not always immediately clear. Currently, MV is applied in a rather generic manner; however, the clinicians have recognized the need for accounting for inter-patient variation [35,36]. VALI due to overstretching of lung parenchyma can be minimized by applying lower tidal volumes [37], but this needs to be achieved without allowing $\mathrm{PaCO}_{2}$ and $\mathrm{pH}$ to rise to dangerous levels (permitted hypercapnea). Sophisticated computer simulation platforms that can accurately represent individual COPD patient characteristics could provide a powerful experimental tool for the development of improved treatment strategies for COPD that address many of the above issues.

\section{Additional file}

Additional file 1: Simulation model description and optimization algorithm description. 


\section{Acknowledgements}

This work was supported from the UK Engineering and Physical Sciences Research Council (EP/F057016/2, EP/F057059/1, EP/1036680/2) and Medical Research Council (G1002017, MR/K019783).

\section{Author details}

${ }^{1}$ School of Engineering, University of Warwick, Coventry CV4 7AL, UK. ${ }^{2}$ Anaesthesia \& Critical Care Research Group, School of Medicine, University of Nottingham, Nottingham NG7 2UH, UK.

Received: 4 April 2014 Accepted: 21 July 2014

Published online: 20 September 2014

\section{References}

1. Kent BD, Mitchell PD, McNicholas WT (2011) Hypoxemia in patients with COPD: cause, effects, and disease progression. Int J Chron Obstruct Pulmon Dis 6:199

2. Murray CJ, Lopez AD (1997) Alternative projections of mortality and disability by cause 1990-2020: Global Burden of Disease Study. Lancet 349(9064):1498-1504

3. Reddy RM, Guntupalli KK (2007) Review of ventilatory techniques to optimize mechanical ventilation in acute exacerbation of chronic obstructive pulmonary disease. Int J Chron Obstruct Pulmon Dis 2(4):441

4. Gutierrez G (2004) A mathematical model of tissue-blood carbon dioxide exchange during hypoxia. Am J Respir Crit Care Med 169(4):525-533

5. Hickling KG (2001) Best compliance during a decremental, but not incremental, positive end-expiratory pressure trial is related to open-lung positive end-expiratory pressure: a mathematical model of acute respiratory distress syndrome lungs. Am J Respir Crit Care Med 163(1):69-78

6. Huo B, Fu R (2012) Recent advances in theoretical models of respiratory mechanics. Acta Mechanica Sinica 28(1):1-7

7. Loeppky JA, Caprihan A, Altobelli SA, Icenogle MV, Scotto P, Vidal Melo MF (2006) Validation of a two-compartment model of ventilation/perfusion distribution. Respir Physiol Neurobiol 151(1):74-92

8. Martin S, Similowski T, Straus C, Maury B (2008) Impact of respiratory mechanics model parameters on gas exchange efficiency. In: ESAIM: Proceedings. Volume 23. EDP Sciences, pp 30-47

9. Steimle KL, Mogensen ML, Karbing DS, Bernardino Dela Serna J, Andreassen S (2011) A model of ventilation of the healthy human lung. Comput Methods Programs Biomed 101(2):144-155

10. Wilson A, Murphy C, Brook B, Breen D, Miles A, Tilley D (2009) A computer model of the artificially ventilated human respiratory system in adult intensive care. Med Eng Phys 31(9):1118-1133

11. Yem JS, Tang Y, Turner MJ, Baker AB (2003) Sources of error in noninvasive pulmonary blood flow measurements by partial rebreathing: a computer model study. Anesthesiology 98(4):881-887

12. Wagner P, Dantzker D, Dueck R, Clausen J, West J (1977) Ventilation-perfusion inequality in chronic obstructive pulmonary disease. J Clin Invest 59(2):203

13. Kathirgamanathan A, McCahon R, Hardman J (2009) Indices of pulmonary oxygenation in pathological lung states: an investigation using high-fidelity, computational modelling. Br J Anaesth 103(2):291-297

14. Yem J, Turner M, Baker A, Young I, Crawford A (2006) A tidally breathing model of ventilation, perfusion and volume in normal and diseased lungs. Br J Anaesth 97(5):718-731

15. Karbing DS, Kjærgaard S, Andreassen S, Espersen K, Rees SE (2011) Minimal model quantification of pulmonary gas exchange in intensive care patients. Med Eng Phys 33(2):240-248

16. Das A, Menon PP, Hardman JG, Bates DG (2013) Optimization of mechanical ventilator settings for pulmonary disease states. IEEE Trans Biomed Eng 60(6):1599-1607

17. Hardman J, Bedforth N, Ahmed A, Mahajan R, Aitkenhead A (1998) A physiology simulator: validation of its respiratory components and its ability to predict the patient's response to changes in mechanical ventilation. $\mathrm{Br} J$ Anaesth 81(3):327-332

18. Wang W, Menon P, Bates D, Bennani S (2010) Robustness analysis of attitude and orbit control systems for flexible satellites. Control Theory Appl IET 4(12):2958-2970

19. Hardman J, Wills J (2006) The development of hypoxaemia during apnoea in children: a computational modelling investigation. Br J Anaesth 97(4):564-570

20. McCahon R, Columb M, Mahajan R, Hardman J (2008) Validation and application of a high-fidelity, computational model of acute respiratory distress syndrome to the examination of the indices of oxygenation at constant lung-state. Br J Anaesth 101(3):358-365

21. Jolliet P, Watremez C, Roeseler J, Ngengiyumva J, De Kock M, Clerbaux T, Tassaux D, Reynaert M, Detry B, Liistro G (2003) Comparative effects of helium-oxygen and external positive end-expiratory pressure on respiratory mechanics, gas exchange, and ventilation-perfusion relationships in mechanically ventilated patients with chronic obstructive pulmonary disease. Intensive Care Med 29(9):1442-1450

22. Loring SH, Garcia-Jacques M, Malhotra A (2009) Pulmonary characteristics in COPD and mechanisms of increased work of breathing. J Appl Physiol 107(1):309-314

23. Glodberg DE (1989) Genetic algorithms in search, optimization, and machine learning. Boston: Addion wesley.

24. Esteban A, Anzueto A, Alia I, Gordo F, Apezteguia C, Palizas F, Cide D, Goldwaser R, Soto L, Bugedo G (2000) How is mechanical ventilation employed in the intensive care unit? An international utilization review. Am J Respir Crit Care Med 161(5):1450-1458

25. Esteban A, Anzueto A, Frutos F, Alia I, Brochard L, Stewart TE, Benito S, Epstein SK, Apezteguia C, Nightingale P, Arroliga AC, Tobin MJ (2002) Characteristics and outcomes in adult patients receiving mechanical ventilation: a 28-day international study. JAMA 287(3):345-355

26. Deb K, Pratap A, Agarwal S, Meyarivan T (2002) A fast and elitist multiobjective genetic algorithm: NSGA-II. Evol Comput IEEE Trans 6(2):182-197 
27. Mannino DM, Buist AS (2007) Global burden of COPD: risk factors, prevalence, and future trends. Lancet 370(9589):765-773

28. Rabe KF, Hurd S, Anzueto A, Barnes PJ, Buist SA, Calverley P, Fukuchi Y, Jenkins C, Rodriguez-Roisin R, Van Weel C, Zielinski J (2007) Global strategy for the diagnosis, management, and prevention of chronic obstructive pulmonary disease: GOLD executive summary. Am J Respir Crit Care Med 176(6):532-555

29. Breen D, Churches T, Hawker F, Torzillo PJ (2002) Acute respiratory failure secondary to chronic obstructive pulmonary disease treated in the intensive care unit: a long term follow up study. Thorax 57(1):29-33

30. Matic I, Danic D, Majeric-Kogler V, Jurjevic M, Mirkovic I, Mrzljak Vucinic N (2007) Chronic obstructive pulmonary disease and weaning of difficult-to-wean patients from mechanical ventilation: randomized prospective study. Croat Med J 48(1):51-58

31. Budweiser S, Jörres RA, Pfeifer M (2008) Treatment of respiratory failure in COPD. Int J Chron Obstruct Pulmon Dis 3(4):605

32. Tawhai MH, Clark AR, Burrowes KS (2011) Computational models of the pulmonary circulation: insights and the move towards clinically directed studies. Pulm Circ 1(2):224-238

33. Ferrer M, Esquinas A, Leon M, Gonzalez G, Alarcon A, Torres A (2003) Noninvasive ventilation in severe hypoxemic respiratory failure: a randomized clinical trial. Am J Respir Crit Care Med 168(12):1438-1444

34. Rodriquez-Roisin R, Drakulovic M, Rodriguez DA, Roca J, Barbera JA, Wagner PD (2009) Ventilation-perfusion imbalance and chronic obstructive pulmonary disease staging severity. J Appl Physiol 106(6):1902-1908

35. Marini JJ (2012) Lower tidal volumes for everyone: principle or prescription? Intensive Care Med 39(1):3-5

36. Fernando F-V, Ferguson ND, Andrés E (2009) Mechanical ventilation: quo vadis? Intensive Care Med 35(5):775-778

37. Tremblay LN, Slutsky AS (2006) Ventilator-induced lung injury: from the bench to the bedside. Intensive Care Med 32(1):24-33

doi:10.1186/s40635-014-0023-0

Cite this article as: Wang et al:: Can computer simulators accurately represent the pathophysiology of individual COPD patients? Intensive Care Medicine Experimental 2014 2:23.

\section{Submit your manuscript to a SpringerOpen ${ }^{\circ}$ journal and benefit from:}

- Convenient online submission

Rigorous peer review

- Immediate publication on acceptance

- Open access: articles freely available online

- High visibility within the field

- Retaining the copyright to your article

Submit your next manuscript at $\boldsymbol{\sim}$ springeropen.com 